\title{
Experimental
}

\section{General Experimental}

Unless otherwise specified, solvents were dried over activated alumina. Triethylamine $\left(\mathrm{NEt}_{3}\right)$ was distilled from $\mathrm{CaH}_{2}$. Dimethylformamide (DMF) was dried over $3 \AA$ molecular sieves for 3 days and then distilled from $\mathrm{P}_{2} \mathrm{O}_{5}$. Analytical thin-layer chromatography (tlc) was run on K6F silica gel 60A and flash column chromatography was run with 230-400 mesh ASTM 60A silica gel. Unless otherwise specified, all reactions were performed with either flame or oven dried glassware under an $\mathrm{N}_{2}$ atmosphere.

7-Bromo-1-(tert-butyldimethylsilanyloxymethyl)-isatin (8). To a dark orange solution of $7^{17}(6 \mathrm{~g}, 26.5 \mathrm{mmol})$ in THF $(130 \mathrm{~mL})$ was added $\mathrm{NaH}(3.2 \mathrm{~g}$ of $60 \%$ suspension in mineral oil, $79.5 \mathrm{mmol}$ ) in one portion at $0{ }^{\circ} \mathrm{C}$. The ensuing deep purple solution containing suspended $\mathrm{NaH}$ was stirred for $15 \mathrm{~min}$, until no $\mathrm{H}_{2}$ evolution had stopped. Freshly prepared chloromethyl tbutyldimethylsilyl ether ${ }^{10}(11.5 \mathrm{~g}, 63.6 \mathrm{mmol})$ was added as a solution in hexanes $(\sim 30 \mathrm{~mL})$ via cannula. The cold bath was then removed and the solution was stirred for 1 day at ambient temperature. The resulting orange/yellow suspension was cooled to $0^{\circ} \mathrm{C}$ and quenched by slow addition of $\mathrm{AcOH}(12 \mathrm{~mL})$. The orange/yellow suspension was then poured into sat'd $\mathrm{NaHCO}_{3}$ solution and extracted with $\mathrm{Et}_{2} \mathrm{O}(3 \mathrm{X})$. The $\mathrm{Et}_{2} \mathrm{O}$ extracts were combined and washed with water $(2 \mathrm{X})$, dried $\left(\mathrm{MgSO}_{4}\right)$, and concentrated to a thick oil. The residue was purified by flash column chromatography on silica gel (12:1 hexanes/acetone) to afford $6 \mathrm{~g}$ (61\% yield) of $\mathbf{8}$ as yellow crystals; analytical tlc, 1:11 ether/hexanes, $\mathrm{Rf}=0.20$. Pure material was obtained by crystallization from hexanes, mp $117-118{ }^{\circ} \mathrm{C}$. ES Molecular ion calcd for 
$\mathrm{C}_{15} \mathrm{H}_{20} \mathrm{BrNO}_{3} \mathrm{SiNa}+$ : 392.0288 ; found $\mathrm{m} / z=392.0276$, error= $3 \mathrm{ppm}$; IR (neat, $\mathrm{cm}^{-1}$ ) $1740(\mathrm{C}=\mathrm{O}), 1605(\mathrm{C}=\mathrm{O}), 1081 .{ }^{1} \mathrm{H} \mathrm{NMR}\left(400 \mathrm{MHz}, \mathrm{CDCl}_{3}, \mathrm{ppm}\right) \delta 7.76(1 \mathrm{H}, \mathrm{d}, J=8.1$ Hz) $7.62(1 \mathrm{H}, \mathrm{d}, J=7.4 \mathrm{~Hz}) 7.04(1 \mathrm{H}, \mathrm{dd}, J=8.1,7.4 \mathrm{~Hz}) 5.67(2 \mathrm{H}, \mathrm{s}) 0.89(9 \mathrm{H}, \mathrm{s}) 0.13$

$(6 \mathrm{H}, \mathrm{s}) .{ }^{13} \mathrm{C}$ NMR $\left(100 \mathrm{MHz}, \mathrm{CDCl}_{3}, \mathrm{ppm}\right) \delta 158.5,147.7,144.2,125.4,124.7,120.9$, 105.4, 64.3, 29.9, 25.8, 18.2, -5.0.

\section{3-[5-Benzyloxy-2-(4-methoxybenzyloxy)-phenyl]-7-bromo-1-(tert-} butyldimethylsilanyloxymethyl)-3-hydroxyoxindole $\quad(9)$. To a solution of 4 benzyloxyphenol $(3.8 \mathrm{~g}, 19 \mathrm{mmol}$, Aldrich) in THF $(50 \mathrm{~mL})$ was added $i \mathrm{PrMgCl}(9.8$ $\mathrm{mL}, 1.94 \mathrm{M}$ sol'n in THF, $19 \mathrm{mmol}$ ) dropwise at $0{ }^{\circ} \mathrm{C}$. After addition was complete the cold bath was removed. Upon warming to ambient temperature, the solution became nearly solid with white ppt. The suspension was concentrated and placed under vacuum (0.1 torr) to ensure THF removal. Once residual solvent was removed, 8 (6.2 g, 16.7 mmol) was added in one portion as a solid. The solids were dissolved in $\mathrm{CH}_{2} \mathrm{Cl}_{2}$ (50 $\mathrm{mL}$ ), initially giving a black solution, and heated to $40{ }^{\circ} \mathrm{C}$ for $8 \mathrm{~h}$. The black color gradually faded over 6-8 $\mathrm{h}$ leaving a light brown solution, which was poured into $1 \mathrm{M}$ $\mathrm{H}_{2} \mathrm{SO}_{4}$ and extracted with $\mathrm{Et}_{2} \mathrm{O}(3 \mathrm{X})$. The organics were combined and washed with water, dried $\left(\mathrm{MgSO}_{4}\right)$, and concentrated. To the residue dissolved in DMF $(80 \mathrm{~mL})$ was added $\mathrm{K}_{2} \mathrm{CO}_{3}(9.2 \mathrm{~g}, 67 \mathrm{mmol})$ and $p$-methoxybenzyl bromide $(10 \mathrm{~g}, 50 \mathrm{mmol})$. The brown suspension was stirred for $12 \mathrm{~h}$ and then poured into water. The water was extracted with $\mathrm{Et}_{2} \mathrm{O}(3 \mathrm{X})$. The combined $\mathrm{Et}_{2} \mathrm{O}$ extracts were washed with water $(2 \mathrm{X})$, dried $\left(\mathrm{MgSO}_{4}\right)$, and concentrated. The residue was purified by flash column chromatography on silica gel (5:1 hexanes/acetone) to afford $11.2 \mathrm{~g}$ (97\% yield) of 9 as a white crystalline solid; analytical tlc, $5: 1$ hexanes/acetone, $\mathrm{Rf}=0.23$. Pure material was 
obtained by crystallization from hexanes, mp $151-156{ }^{\circ} \mathrm{C}$. ES Molecular ion calcd for $\mathrm{C}_{36} \mathrm{H}_{40} \mathrm{BrNO}_{6} \mathrm{SiNa}+$ : 714.1700 ; found $\mathrm{m} / z=714.1735$, error= $5 \mathrm{ppm}$; IR (neat, $\mathrm{cm}^{-1}$ ) $3382(\mathrm{OH}), 1739,1723,1465 .{ }^{1} \mathrm{H}$ NMR $\left(400 \mathrm{MHz}, \mathrm{CDCl}_{3}, \mathrm{ppm}\right) \delta 7.52(1 \mathrm{H}, \mathrm{d}, J=3$ Hz) 7.46-7.30 (6H, m) 6.99-6.95 (3H, m) 6.89-6.76 (5H, m) $5.07(2 \mathrm{H}, \mathrm{s}) 4.93(2 \mathrm{H}, \mathrm{ABq}$, $J=10 \mathrm{~Hz}) 4.64(2 \mathrm{H}, \mathrm{ABq}, J=10.2 \mathrm{~Hz}) 3.83(3 \mathrm{H}, \mathrm{s}) 3.01(1 \mathrm{H}, \mathrm{br} \mathrm{s}) 0.85(9 \mathrm{H}, \mathrm{s}) 0.09(3 \mathrm{H}$, s) $0.02(3 \mathrm{H}, \mathrm{s}) .{ }^{13} \mathrm{C} \mathrm{NMR}\left(100 \mathrm{MHz}, \mathrm{CDCl}_{3}, \mathrm{ppm}\right) \delta 177.1,159.5,153.0,149.1,140.7$, 137.1, 135.2, 133.1, 129.7, 129.2, 128.6, 128.0, 127.9, 127.6, 124.0, 123.4, 115.0, 114.1, 114.0, 112.5, 103.0, 77.2, 75.2, 70.7, 70.4, 63.9, 55.2, 25.7, -4.9, -5.4.

\section{3-[5-Benzyloxy-2-(4-methoxybenzyloxy)-phenyl]-7-bromo-1-(tert-}

butyldimethylsilanyloxymethyl)-oxindole (11). To a solution of $9(11.1 \mathrm{~g}, 16 \mathrm{mmol})$ and $\operatorname{EtN}(i \mathrm{Pr})_{2}(8.4 \mathrm{~mL}, 6.2 \mathrm{~g}, 48.2 \mathrm{mmol})$ in $\mathrm{CH}_{2} \mathrm{Cl}_{2}(150 \mathrm{~mL})$ was added freshly distilled thionyl chloride $(1.4 \mathrm{~mL}, 2.28 \mathrm{~g}, 19.2 \mathrm{mmol})$ dropwise over 5 min at $0{ }^{\circ} \mathrm{C}$. During addition the solution initially became a deepening yellow and gradually turned dark brown. The solution was stirred for $10 \mathrm{~min}$ and poured into sat'd $\mathrm{NaHCO}_{3}$ solution at which time the mixtured lightened in color. The aqueous layer was extracted with $\mathrm{Et}_{2} \mathrm{O}$ $(3 \mathrm{X})$. The combined $\mathrm{Et}_{2} \mathrm{O}$ extracts were dried $\left(\mathrm{MgSO}_{4}\right)$ and concentrated to give a yellow foaming gum, which was subsequently dried under vacuum (0.1 torr) for $10 \mathrm{~min}$. To the residual gum dissolved in THF $(140 \mathrm{~mL})$ and $\mathrm{AcOH}(14 \mathrm{~mL})$ was added at $0{ }^{\circ} \mathrm{C}$ Zn dust (31.5 g, $482 \mathrm{mmol},<10$ micron) in one portion and with vigorous stirring. The cold bath was removed after $1 \mathrm{~h}$ and the suspension was stirred for $3 \mathrm{~h}$. The reaction was then checked by ESMS for the appearance of $\mathbf{1 1}\left(+\mathrm{Na}^{+}, 698.2\right)$ and the disappearance of $\mathbf{1 0}\left(+\mathrm{Na}^{+}, 732.1\right)$. If conversion of chloride $\mathbf{1 0}$ was not complete, the suspension was heated at $35{ }^{\circ} \mathrm{C}$ until no starting material was detected (usually $\sim 3 \mathrm{~h}$ ). The suspension 
was then filtered through a cake of Celite and the cake washed with copious amounts of $\mathrm{Et}_{2} \mathrm{O}$ and water. The water layer was washed with $\mathrm{Et}_{2} \mathrm{O}(2 \mathrm{X})$. The combined organics were washed with a minimal amount of water and then sat'd $\mathrm{NaHCO}_{3}$ solution $(2 \mathrm{X})$, dried $\left(\mathrm{MgSO}_{4}\right)$ and concentrated to a yellow/orange solid. The solid was triturated with $\mathrm{Et}_{2} \mathrm{O}(2 \mathrm{X})$ to give $8.6 \mathrm{~g}$ of $\mathbf{1 1}$ as a white solid. The $\mathrm{Et}_{2} \mathrm{O}$ washings were combined and concentrated to a thick orange oil. The residual oil was purified by flash column chromatography on silica gel (5:1 hexanes/ $\left.\mathrm{Et}_{2} \mathrm{O}\right)$ to afford $1.2 \mathrm{~g}(91 \%$ yield total) of $\mathbf{1 1}$ as a white solid; analytical tlc, 5:1 hexanes/Et ${ }_{2} \mathrm{O}, \mathrm{Rf}=0.16$. Pure material was obtained by crystallization from $\mathrm{Et}_{2} \mathrm{O}, \mathrm{mp}$ 159-160 ${ }^{\circ} \mathrm{C}$. ES Molecular ion calcd for $\mathrm{C}_{36} \mathrm{H}_{40} \mathrm{BrNO}_{5} \mathrm{SiNa}+: \quad 698.1751$; found $\mathrm{m} / z=698.1781$, error $=4 \mathrm{ppm}$; IR (neat, $\mathrm{cm}^{-1}$ ) 2917, $1727(\mathrm{C}=\mathrm{O})$, 1231. ${ }^{1} \mathrm{H}$ NMR $\left(500 \mathrm{MHz}, \mathrm{CDCl}_{3}, \mathrm{ppm}\right) \delta$ 7.42-7.30 (6H, m) 7.00$6.78(9 \mathrm{H}, \mathrm{m}) 5.29(1 \mathrm{H}, \mathrm{br} \mathrm{s}) 5.05(1 \mathrm{H}, \mathrm{br} \mathrm{s}) 5.00(2 \mathrm{H}, \mathrm{s}) 4.79-4.54(3 \mathrm{H}, \mathrm{m}) 3.82(3 \mathrm{H}, \mathrm{s})$ $0.89(9 \mathrm{H}, \mathrm{s}) 0.12(3 \mathrm{H}, \mathrm{s}) 0.06(3 \mathrm{H}, \mathrm{s}) .{ }^{13} \mathrm{C} \mathrm{NMR}\left(100 \mathrm{MHz}, \mathrm{CDCl}_{3}, \mathrm{ppm}\right) \delta 176.5,159.3$, $152.8,150.6,140.4,137.0,133.4,132.1,129.4,129.3,128.5,128.4,128.0,127.5,126.4$, $123.5,122.9,114.5,113.9,113.0,102.6,70.7,70.5,64.1,55.2,29.7,25.7,18.1,-5.1$, 5.2 .

\section{3-[5-Benzyloxy-2-(4-methoxybenzyloxy)-phenyl]-7-bromo-1-(tert-} butyldimethylsilanyloxymethyl)-oxindole-3-carboxylic acid methyl ester (12). To a solution of 11 (8.5 g, $12.6 \mathrm{mmol})$ in THF (125 mL) was added $\mathrm{NaH}(1.5 \mathrm{~g}$ of 60\% dispersion in mineral oil, $37.8 \mathrm{mmol}$ ) at $0{ }^{\circ} \mathrm{C}$. After the $\mathrm{H}_{2}$ evolution ceased, the yellow suspension was stirred for $10 \mathrm{~min}$ and then methyl cyanoformate $(5 \mathrm{~mL}, 5.36 \mathrm{~g}, 63$ mmol) was added in one portion. The yellow suspension was heated to $33{ }^{\circ} \mathrm{C}$ for one day, after which time the color had become orange. The mixture was cooled to $0{ }^{\circ} \mathrm{C}$ and 
slowly quenched with $\mathrm{AcOH}$. The ensuing yellow/orange suspension was poured into sat'd $\mathrm{NaHCO}_{3}$ solution and extracted with $\mathrm{Et}_{2} \mathrm{O}(3 \mathrm{X})$. The $\mathrm{Et}_{2} \mathrm{O}$ was then dried $\left(\mathrm{MgSO}_{4}\right)$ and concentrated to a thick oil. The residue was purified by flash column chromatography on silica gel $\left(3: 1\right.$ hexanes/Et ${ }_{2} \mathrm{O}$ to $3: 2$ hexanes/Et $\left.t_{2} \mathrm{O}\right)$ to afford $8.9 \mathrm{~g}$ (97\% yield) of $\mathbf{1 2}$ as a white solid; analytical tlc, 5:1 hexanes/acetone, $\mathrm{Rf}=0.30, \mathrm{mp} 112$ ${ }^{\circ} \mathrm{C}$ (hexanes/acetone). ES Molecular ion calcd for $\mathrm{C}_{38} \mathrm{H}_{42} \mathrm{BrNO}_{7} \mathrm{SiNa}+$ : 756.1806 ; found $m / z=756.1796$, error= $1 \mathrm{ppm}$; IR (neat, $\left.\mathrm{cm}^{-1}\right)$ 2952, 1737, 1227; ${ }^{1} \mathrm{H}$ NMR $(500 \mathrm{MHz}$, $\left.\mathrm{CDCl}_{3}, \mathrm{ppm}\right) \delta 7.46(1 \mathrm{H}, \mathrm{dd}, J=1.2,8.0 \mathrm{~Hz}) 7.37-7.27(8 \mathrm{H}, \mathrm{m}) 6.95-6.85(5 \mathrm{H}, \mathrm{m}) 6.56$ $(1 \mathrm{H}, \mathrm{d}, J=2.9 \mathrm{~Hz}) 5.43(2 \mathrm{H}, \mathrm{ABq}, J=10 \mathrm{~Hz}) 4.96-4.86(4 \mathrm{H}, \mathrm{m}) 3.82(3 \mathrm{H}, \mathrm{s}) 3.39(3 \mathrm{H}, \mathrm{s})$ $0.87(9 \mathrm{H}, \mathrm{s}) 0.08(3 \mathrm{H}, \mathrm{s}) 0.06(3 \mathrm{H}, \mathrm{s}) .{ }^{13} \mathrm{C} \mathrm{NMR}\left(100 \mathrm{MHz}, \mathrm{CDCl}_{3}, \mathrm{ppm}\right) \delta$ 172.6, 168.4, $159.3,152.7,151.2,140.3,136.9,134.9,130.5,129.6,128.6,127.9,127.5,126.9,124.9$, 124.1, 116.6, 114.6, 114.2, 113.7, 102.9, 77.2, 71.2, 70.6, 64.5, 62.0, 55.2, 52.9, 25.7, $18.0,-5.15,-5.2$.

3-[5-Benzyloxy-2-(4-methoxybenzyloxy)-phenyl]-7-bromooxindole-3-carboxylic acid methyl ester (13). To a solution of $12(8.8 \mathrm{~g}, 12 \mathrm{mmol})$ in DMF (45 mL) was added TAS-F (6.61g, $24 \mathrm{mmol}$, Aldrich) as a solution in DMF (45 mL). The clear, slightly yellow solution was stirred for $1 \mathrm{~h}$ and poured into $1 \mathrm{M} \mathrm{H}_{2} \mathrm{SO}_{4}$. The mixture was extracted with $\mathrm{Et}_{2} \mathrm{O}(3 \mathrm{X})$. The $\mathrm{Et}_{2} \mathrm{O}$ was washed with water $(2 \mathrm{X})$, dried $\left(\mathrm{MgSO}_{4}\right)$, and concentrated to a thick oil. The residue was purified by flash column chromatography on silica gel (3:1 hexanes/acetone) to afford $7.06 \mathrm{~g}$ (quantitative yield) of $\mathbf{1 3}$ as a white crystalline solid; analytical tlc, 3:1 hexanes/acetone, $\mathrm{Rf}=0.19, \mathrm{mp} 173-175{ }^{\circ} \mathrm{C}$ (hexanes/acetone). ES Molecular ion calcd for $\mathrm{C}_{31} \mathrm{H}_{26} \mathrm{BrNO}_{6} \mathrm{Na}+$ : 610.0836 ; found $\mathrm{m} / \mathrm{z}=$ 610.0840, error= 1 ppm; IR (neat, $\left.\mathrm{cm}^{-1}\right) 3200(\mathrm{NH}), 1723(\mathrm{C}=\mathrm{O}), 1495 .{ }^{1} \mathrm{H}$ NMR (500 
$\left.\mathrm{MHz}, \mathrm{CDCl}_{3}, \mathrm{ppm}\right) \delta$ 7.37-7.27 (9H, m) $7.23(1 \mathrm{H}, \mathrm{d}, J=7.5 \mathrm{~Hz}) 6.94(1 \mathrm{H}, \mathrm{t}, J=9 \mathrm{~Hz})$

6.91-6.87 (4H, m) $6.67(1 \mathrm{H}, \mathrm{d}, J=2.5 \mathrm{~Hz}) 4.95(2 \mathrm{H}, \mathrm{ABq}, J=10.5 \mathrm{~Hz}) 4.91(2 \mathrm{H}, \mathrm{ABq}$, $J=10.5 \mathrm{~Hz}) 3.82(3 \mathrm{H}, \mathrm{s}) 3.49(3 \mathrm{H}, \mathrm{s}) .{ }^{13} \mathrm{C} \mathrm{NMR}\left(125 \mathrm{MHz}, \mathrm{CDCl}_{3}, \mathrm{ppm}\right) \delta 172.5,168.3$, $159.4,152.7,151.1,140.3,136.8,131.7,129.6,129.5,128.5,128.4,128.0,127.5,126.3$, $124.9,124.0,116.6,114.7,114.1,113.8,102.5,71.3,70.6,63.9,55.3,53.1$.

\section{3-[5-Benzyloxy-2-(4-methoxybenzyloxy)-phenyl]-7-bromo-2-oxo-2,3-dihydroindole-} 1,3-dicarboxylic acid 1-allyl ester 3-methyl ester (14). To a solution of $\mathbf{1 3}$ (2.5 g, 4.25 mmol) in THF (15 mL) was added NaH (306 mg of 60\% dispersion in mineral oil, 12.75 mmol) at $0{ }^{\circ} \mathrm{C}$. The suspension was then warmed to rt, stirred for $10 \mathrm{~min}$ and then cooled to $0{ }^{\circ} \mathrm{C}$. Freshly distilled methyl chloroformate $(0.68 \mathrm{~mL}, 768 \mathrm{mg}, 6.37 \mathrm{mmol}$, Aldrich) was added as a solution in $\mathrm{CH}_{2} \mathrm{Cl}_{2}(7 \mathrm{~mL})$. A white ppt formed during addition. The suspension was then warmed to rt (water bath) and DMAP (312 $\mathrm{mg}, 2.55 \mathrm{mmol})$ was added as a solution in $\mathrm{CH}_{2} \mathrm{Cl}_{2}(7 \mathrm{~mL})$. A yellow color developed with every drop but quickly faded. After addition was complete the reaction was stirred for $10 \mathrm{~min}$ and then quenched with $\mathrm{AcOH}$ at $0{ }^{\circ} \mathrm{C}$. The mixture was poured into sat'd $\mathrm{NaHCO}_{3}$ solution and extracted with $\mathrm{Et}_{2} \mathrm{O}(3 \mathrm{X})$. The $\mathrm{Et}_{2} \mathrm{O}$ was washed with $1 \mathrm{M} \mathrm{H}_{2} \mathrm{SO}_{4}$, sat'd $\mathrm{NaHCO}_{3}$ solution, and then dried $\left(\mathrm{MgSO}_{4}\right)$ and concentrated. The residue was purified by flash column chromatography on silica gel $\left(1: 1\right.$ hexanes/ $\left.\mathrm{Et}_{2} \mathrm{O}\right)$ to afford $2.82 \mathrm{~g}$ (99\% yield) of 14 as a white foam; analytical tlc, 1:1 hexanes/acetone, $\mathrm{Rf}=0.33$. ES Molecular ion calcd for $\mathrm{C}_{35} \mathrm{H}_{30} \mathrm{BrNO} \mathrm{N}_{8} \mathrm{Na}+$ : 696.1047 ; found $\mathrm{m} / z=696.1040$, error $=1 \mathrm{ppm}$; IR (neat, $\mathrm{cm}^{-1}$ ) $1794(\mathrm{C}=\mathrm{O}), 1744(\mathrm{C}=\mathrm{O}), 1495 .{ }^{1} \mathrm{H}$ NMR $\left(500 \mathrm{MHz}, \mathrm{CDCl}_{3}, \mathrm{ppm}\right) \delta 7.52(1 \mathrm{H}, \mathrm{dd}, J=$ 8.0, 1.0 Hz) 7.37-7.29 (5H, m) 7.27 (1H, dd, 7.6, $1.0 \mathrm{~Hz}) 7.25-7.22(2 \mathrm{H}, \mathrm{m}) 7.03(1 \mathrm{H}, \mathrm{t}$, $J=8.0 \mathrm{~Hz}) 6.90-6.83(4 \mathrm{H}, \mathrm{m}) 6.65(1 \mathrm{H}, \mathrm{d}, J=2.4 \mathrm{~Hz}) 6.01(1 \mathrm{H}, \mathrm{ddt}, J=17.1,10.5,5.9$ 
Hz) $5.44(1 \mathrm{H}, \mathrm{dd}, J=17.1,1.2 \mathrm{~Hz}) 5.30(1 \mathrm{H}, \mathrm{dd}, J=10.5,1.2 \mathrm{~Hz}) 4.97-4.87(6 \mathrm{H}, \mathrm{m}) 3.79$

$(3 \mathrm{H}, \mathrm{s}) 3.45(3 \mathrm{H}, \mathrm{s}) .{ }^{13} \mathrm{C} \mathrm{NMR}\left(100 \mathrm{MHz}, \mathrm{CDCl}_{3}, \mathrm{ppm}\right) \delta 170.3,167.7,159.2,152.7$, $150.9,149.3,138.7,136.8,134.1,130.6,130.2,129.3,128.6,128.5,128.0,127.5,126.1$, 125.9, 124.8, 119.9, 116.7, 115.0, 114.3, 113.7, 106.8, 71.1, 70.7, 69.0, 63.1, 55.2, 53.3.

6-Benzyloxy-1-bromo-9-oxa-10-azaindeno[1,2-a]indene-4b,10-dicarboxylic acid 10allyl ester 4b-methyl ester (16). $\mathrm{NaBH}_{4}(561 \mathrm{mg}, 14.8 \mathrm{mmol})$ was stirred in $\mathrm{MeOH}(20$ $\mathrm{mL}$ ) at $0{ }^{\circ} \mathrm{C}$ until no solid was visually observed. The resulting clear, colorless solution that vigorously evolved $\mathrm{H}_{2}$ was quickly added to a solution of $\mathbf{1 4}(2.5 \mathrm{~g}, 3.7 \mathrm{mmol})$ in THF $(20 \mathrm{~mL})$ at $0{ }^{\circ} \mathrm{C}$. The reaction was stirred for $10 \mathrm{~min}$ and then poured into a rapidly stirred solution of $1 \mathrm{M} \mathrm{H}_{2} \mathrm{SO}_{4}$. After foaming subsided, the mixture was extracted with $\mathrm{Et}_{2} \mathrm{O}(3 \mathrm{X})$. The $\mathrm{Et}_{2} \mathrm{O}$ was washed with sat'd $\mathrm{NaHCO}_{3}$ solution, dried $\left(\mathrm{MgSO}_{4}\right)$, and concentrated. The residue was placed under vacuum ( 0.1 torr) for 30 min to remove residual solvents. To a solution of the residue and $\mathrm{NEt}_{3}(1.55 \mathrm{~mL}, 1.12 \mathrm{~g}, 11.1 \mathrm{mmol})$ in $\mathrm{CH}_{2} \mathrm{Cl}_{2}(40 \mathrm{~mL})$ was added $\mathrm{Ms}_{2} \mathrm{O}\left(967 \mathrm{mg}, 5.55 \mathrm{mmol}\right.$, Aldrich) in one portion at $0{ }^{\circ} \mathrm{C}$. The yellow solution was stirred at $0{ }^{\circ} \mathrm{C}$ until all of the $\mathrm{Ms}_{2} \mathrm{O}$ had dissolved and the cold bath was then removed. After stirring for $6 \mathrm{~h}$, the mixture was poured into $5 \% \mathrm{NaHCO}_{3}$ solution and extracted with $\mathrm{Et}_{2} \mathrm{O}(3 \mathrm{X})$. The $\mathrm{Et}_{2} \mathrm{O}$ was dried $\left(\mathrm{MgSO}_{4}\right)$, concentrated and the residue was purified by flash column chromatography on silica gel (2:1 hexanes/ $\mathrm{Et}_{2} \mathrm{O}$ ) to afford $1.76 \mathrm{~g}$ (93\% yield) of $\mathbf{1 6}$ as a white solid; analytical tlc, 5:1 hexanes/acetone, $\mathrm{Rf}=0.25$, mp $108{ }^{\circ} \mathrm{C} \quad\left(\mathrm{Et}_{2} \mathrm{O}\right)$. ES Molecular ion calcd for $\mathrm{C}_{27} \mathrm{H}_{22} \mathrm{BrNO}_{6} \mathrm{Na}+$ : 558.0523 ; found $\mathrm{m} / \mathrm{z}=558.0515$, error= $1 \mathrm{ppm}$; IR (neat, $\mathrm{cm}^{-1}$ ) 1737 $(\mathrm{C}=\mathrm{O}), 1486,1192 .{ }^{1} \mathrm{H} \mathrm{NMR}\left(500 \mathrm{MHz}, \mathrm{CDCl}_{3}, \mathrm{ppm}\right) \delta 7.48(1 \mathrm{H}, \mathrm{dd}, J=8.0,1.0 \mathrm{~Hz})$ 7.44-7.31 (6H, m) $7.28(1 \mathrm{H}, \mathrm{d}, J=2.7 \mathrm{~Hz}) 7.16(1 \mathrm{H}, \mathrm{s}) 6.99(1 \mathrm{H}, \mathrm{t}, J=7.8 \mathrm{~Hz}) 6.80(1 \mathrm{H}$, 
dd, $J=8.8,2.7 \mathrm{~Hz}), 6.74(1 \mathrm{H}, \mathrm{d}, J=8.8 \mathrm{~Hz}) 6.05(1 \mathrm{H}, \mathrm{ddt}, J=17.1,10.5,5.8 \mathrm{~Hz}) 5.43$ $(1 \mathrm{H}, \mathrm{ddt}, J=17.1,1.5,1.2 \mathrm{~Hz}) 5.31(1 \mathrm{H}, \mathrm{ddt}, J=10.5,1.5,1.2 \mathrm{~Hz}) 5.01(2 \mathrm{H}, \mathrm{s}) 4.85(2 \mathrm{H}$, $\mathrm{ABq}[J=13.2 \mathrm{~Hz}]$ of dt $[J=5.8,1.2 \mathrm{~Hz}]) 3.82(3 \mathrm{H}, \mathrm{s}) .{ }^{13} \mathrm{C} \mathrm{NMR}\left(125 \mathrm{MHz}, \mathrm{CDCl}_{3}\right.$, ppm) $\delta 168.5,153.9,152.3,152.2,139.9,136.8,134.5,134.3,131.6,128.6,128.0,127.6$, $126.9,126.1,123.3,119.0,116.0,112.1,111.8,110.3,100.8,71.1,67.7,64.4,53.5$.

6-Benzyloxy-1-bromo-9-oxa-10-azaindeno[1,2-a]indene-4b,10-dicarboxylic acid 10allyl ester (17). To a solution of 16 (1.03 g, $1.97 \mathrm{mmol})$ in THF (6.6 mL) and $\mathrm{MeOH}(10$ $\mathrm{mL})$ was added $\mathrm{NaOH}(304 \mathrm{mg}, 7.6 \mathrm{mmol})$ as a solution in water $(3.3 \mathrm{~mL})$. The slightly yellow solution was stirred for $30 \mathrm{~min}$, poured into $1 \mathrm{M} \mathrm{H}_{2} \mathrm{SO}_{4}$, and extracted with $\mathrm{Et}_{2} \mathrm{O}$ ( $3 \mathrm{X})$. The $\mathrm{Et}_{2} \mathrm{O}$ was washed with water, dried $\left(\mathrm{MgSO}_{4}\right)$, and concentrated to a thick residue. The residue was dissolved in a minimal amount of $\mathrm{CH}_{2} \mathrm{Cl}_{2}(0.2 \mathrm{~mL})$ and diluted with $\mathrm{Et}_{2} \mathrm{O}(2 \mathrm{~mL})$ and hexanes $(0.5 \mathrm{~mL})$. The solution was placed in the freezer $\left(-20^{\circ} \mathrm{C}\right)$ overnight. The supernatant was then removed by cannula at $-78{ }^{\circ} \mathrm{C}$ and the remaining crystals were washed with $\mathrm{Et}_{2} \mathrm{O}(0.8 \mathrm{~mL})$ at $-78{ }^{\circ} \mathrm{C}$. The crystals were collected by removing the $\mathrm{Et}_{2} \mathrm{O}$ via cannula and placing under vacuum ( 0.1 torr). The supernatant was concentrated to a thick residue which was subjected to this crystallization procedure to afford another crop of crystals giving a total of $996 \mathrm{mg}$ (99\% yield) of $\mathbf{1 7}$ in 2 crops as white crystals; mp $144-145{ }^{\circ} \mathrm{C}$. ES Molecular ion calcd for $\mathrm{C}_{26} \mathrm{H}_{20} \mathrm{BrNO}_{6} \mathrm{Na}+$ : 544.0366 ; found $m / z=544.0359$, error= $1 \mathrm{ppm}$; IR (neat, $\left.\mathrm{cm}^{-1}\right) 3066(\mathrm{COOH}), 1715(\mathrm{C}=\mathrm{O}), 1731$ $(\mathrm{C}=\mathrm{O}) .{ }^{1} \mathrm{H} \mathrm{NMR}\left(400 \mathrm{MHz}, \mathrm{CDCl}_{3}, \mathrm{ppm}\right) \delta 11.82(1 \mathrm{H}, \mathrm{s}) 7.48(1 \mathrm{H}, \mathrm{dd}, J=8.0,1.1 \mathrm{~Hz})$ 7.42-7.28 (7H, m) $7.12(1 \mathrm{H}, \mathrm{s}) 6.98(1 \mathrm{H}, \mathrm{t}, J=8.0 \mathrm{~Hz}) 6.80(1 \mathrm{H}, \mathrm{dd}, J=8.8,2.6 \mathrm{~Hz}) 6.74$ $(1 \mathrm{H}, \mathrm{d}, J=8.8 \mathrm{~Hz}) 6.04(1 \mathrm{H}, \mathrm{ddt}, J=17.2,10.5,5.9 \mathrm{~Hz}) 5.42(1 \mathrm{H}, \mathrm{ddt}, J=17.2,1.5,1.1$ Hz) $5.30(1 \mathrm{H}$, ddt, $J=10.5,1.5,1.1 \mathrm{~Hz}) 5.00(2 \mathrm{H}, \mathrm{s}) 4.85(2 \mathrm{H}, \mathrm{m}) .{ }^{13} \mathrm{C}$ NMR $(100 \mathrm{MHz}$, 
$\left.\mathrm{CDCl}_{3}, \mathrm{ppm}\right) \delta 173.4,154.0,152.6,152.2,139.8,136.7,134.7,133.7,131.5,128.6$, 128.0, 127.6, 127.0, 125.4, 123.4, 119.1, 116.4, 112.2, 111.7, 110.5, 100.5, 71.1, 67.9, 64.3.

\section{6-Benzyloxy-1-trimethylstannanyl-9-oxa-10-azaindeno[1,2-a]indene-4b,10-}

dicarboxylic acid 10-allyl ester 4b-methyl ester (18). To a solution of 17 (980 mg, $1.927 \mathrm{mmol})$ in THF (20 mL) was added $\mathrm{NaH}(268 \mathrm{mg}$ of $60 \%$ dispersion in mineral oil, $6.7 \mathrm{mmol}$, stored in glove box) at $0{ }^{\circ} \mathrm{C}$. The suspension was stirred for $10 \mathrm{~min}$ and then cooled to $-78{ }^{\circ} \mathrm{C}$. Freshly titrated $n \mathrm{BuLi}(1.2 \mathrm{~mL}, 2.02 \mathrm{mmol}, 1.65 \mathrm{M}$ sol'n in hexanes) was then slowly added down the side of the flask to prevent exotherms. The solution became yellow upon addition and was stirred for 2 min. A freshly dried solution (mol. sieves, THF) of $\mathrm{ClSnMe}_{3}(997 \mathrm{mg}, 5 \mathrm{mmol})$ in THF $(4.7 \mathrm{~mL})$ was added and the cold bath was removed. Once at rt the solution was poured into $0.1 \mathrm{M} \mathrm{H}_{2} \mathrm{SO}_{4}$ and extracted with $\mathrm{Et}_{2} \mathrm{O}(3 \mathrm{X})$. The $\mathrm{Et}_{2} \mathrm{O}$ was then washed with water, dried $\left(\mathrm{MgSO}_{4}\right)$, and concentrated to a thick oil. The residue was dissolved in $\mathrm{MeOH}(5 \mathrm{~mL})$ and benzene (15 $\mathrm{mL})$ and treated with $\mathrm{TMSCHN}_{2}$ dropwise until a yellow color persisted $(\sim 2 \mathrm{~mL}, 2.0 \mathrm{M}$ sol'n). The yellow solution was then poured into $1 \mathrm{M} \mathrm{H}_{2} \mathrm{SO}_{4}$ and extracted with $\mathrm{Et}_{2} \mathrm{O}$ (3 $\mathrm{X})$. The $\mathrm{Et}_{2} \mathrm{O}$ was dried $\left(\mathrm{MgSO}_{4}\right)$, and concentrated. The residue was purified by flash column chromatography on silica gel (10:1 hexanes/acetone) to afford $759 \mathrm{mg}(67 \%$ yield) of $\mathbf{1 8}$ as a white foam; analytical tlc, 5:1 hexanes/acetone, $\mathrm{Rf}=0.45$. Pure material was obtained by crystallization from $\mathrm{Et}_{2} \mathrm{O} / \mathrm{hexanes,} \mathrm{mp} 104-106{ }^{\circ} \mathrm{C}$. ES Molecular ion calcd for $\mathrm{C}_{30} \mathrm{H}_{31} \mathrm{NO}_{6} \mathrm{SnNa+}$ : 644.1066 ; found $m / z=644.1052$, error= $2 \mathrm{ppm}$; IR (neat,

$\left.\mathrm{cm}^{-1}\right) 1737(\mathrm{C}=\mathrm{O}), 1715(\mathrm{C}=\mathrm{O}), 1486 .{ }^{1} \mathrm{H}$ NMR $\left(500 \mathrm{MHz}, \mathrm{CDCl}_{3}, \mathrm{ppm}\right) \delta 7.50(1 \mathrm{H}, \mathrm{dd}$, $J=7.6,1.2 \mathrm{~Hz}) 7.46(1 \mathrm{H}, \mathrm{dd}, J=7.3,1.2 \mathrm{~Hz}) 7.43-7.31(5 \mathrm{H}, \mathrm{m}) 7.29(1 \mathrm{H}, \mathrm{d}, J=2.4 \mathrm{~Hz})$ 
$7.22(1 \mathrm{H}, \mathrm{s}) 7.07(1 \mathrm{H}, \mathrm{t}, J=7.4 \mathrm{~Hz}) 6.78(1 \mathrm{H}, \mathrm{dd}, J=8.8,2.7 \mathrm{~Hz}) 6.74(1 \mathrm{H}, \mathrm{d}, J=8.8 \mathrm{~Hz})$

$6.05(1 \mathrm{H}, \mathrm{ddt}, J=17.2,10.2,5.6 \mathrm{~Hz}) 5.46(1 \mathrm{H}, \mathrm{ddt}, J=17.2,1.5,1.2 \mathrm{~Hz}) 5.32(1 \mathrm{H}, \mathrm{ddt}$, $J=10.2,1.5,1.2 \mathrm{~Hz}) 5.01(2 \mathrm{H}, \mathrm{s}) 4.82(2 \mathrm{H}, \mathrm{d}, J=5.6 \mathrm{~Hz}) 3.81(3 \mathrm{H}, \mathrm{s}) 0.24(9 \mathrm{H}, \mathrm{s}$, $\left.{ }^{1} \mathrm{~J}_{\mathrm{SnH}} \mathrm{av}=27.2 \mathrm{~Hz}\right) .{ }^{13} \mathrm{C} \mathrm{NMR}\left(125 \mathrm{MHz}, \mathrm{CDCl}_{3}, \mathrm{ppm}\right) \delta 169.2,153.9,153.5,152.2$, $146.2,138.2,136.9,131.9,131.8,129.6,128.6,128.0,127.6,127.0,124.5,124.2,118.7$, $115.8,111.8,110.2,98.4,71.1,67.1,63.5,53.3,-5.7\left({ }^{1} \mathrm{~J}_{\mathrm{SnC}} \mathrm{av}=185.8 \mathrm{~Hz}\right)$.

\section{6-Benzyloxy-1-trimethylstannanyl-9a,10-dihydro-9-oxa-10-azaindeno[1,2-a]indene-}

4b-carboxylic acid methyl ester (19). The method of Guibe was followed with slight modification. ${ }^{12}$ To a solution of $\mathbf{1 8}(540 \mathrm{mg}, 0.87 \mathrm{mmol})$ and 1,3-dimethylbarbituric acid (428 mg, $2.74 \mathrm{mmol})$ in $\mathrm{CH}_{2} \mathrm{Cl}_{2}(9 \mathrm{~mL})$ was added $\mathrm{Pd}\left(\mathrm{PPh}_{3}\right)_{4}(40 \mathrm{mg}, 0.0348 \mathrm{mmol})$ in one portion at $0{ }^{\circ} \mathrm{C}$. The solution became yellow as the catalyst dissolved and then became clear and colorless. After $\sim 5$ min the yellow color reappeared, signifying the completion of the reaction. The yellow solution was poured into 1:1 50\% sat'd $\mathrm{NaHCO}_{3}$ solution $/ 50 \%$ sat'd $\mathrm{Na}_{2} \mathrm{CO}_{3}$ solution and extracted with $\mathrm{Et}_{2} \mathrm{O}(3 \mathrm{X})$. The $\mathrm{Et}_{2} \mathrm{O}$ was washed with water, dried over $\mathrm{MgSO}_{4}$ and then directly filtered through a plug of silica, washing several times with $\mathrm{Et}_{2} \mathrm{O}$. The $\mathrm{Et}_{2} \mathrm{O}$ was concentrated and the residue was purified by flash column chromatography on silica gel (9.5:1 hexanes/EtOAc) to afford $307 \mathrm{mg}$ (66\% yield) of $\mathbf{1 9}$ as a white foam contaminated with $<10 \%$ of a Me3Sncontaining impurity. 5,5-Diallyl-1,3-dimethylbarbituric acid eluted in slightly later fractions, which yellowed upon standing; analytical tlc, 5:1 hexanes/acetone, $\mathrm{Rf}=0.50$. ES Molecular ion calcd for $\mathrm{C}_{26} \mathrm{H}_{27} \mathrm{NO}_{4} \mathrm{SnNa}+$ : 560.0854 ; found $m / z=560.0840$, error $=2$

ppm; IR (neat, $\left.\mathrm{cm}^{-1}\right) 3412(\mathrm{NH}), 1733(\mathrm{C}=\mathrm{O}), 1485 .{ }^{1} \mathrm{H}$ NMR (500 MHz, $\left.\mathrm{CDCl}_{3}, \mathrm{ppm}\right) \delta$ 7.49-7.15 (8H, m) 6.84-6.69 (4H, m) $5.00(2 \mathrm{H}, \mathrm{s}) 4.89(1 \mathrm{H}, \mathrm{d}, J=2.4 \mathrm{~Hz}) 3.80(3 \mathrm{H}, \mathrm{s})$ 
$0.33\left(9 \mathrm{H}, \mathrm{s}, \mathrm{J}_{\mathrm{SnH}} \mathrm{av}=27.3 \mathrm{~Hz}\right) .{ }^{13} \mathrm{C} \mathrm{NMR}\left(100 \mathrm{MHz}, \mathrm{CDCl}_{3}, \mathrm{ppm}\right) \delta 170.1,153.6,153.3$, $152.6,137.1,136.8,128.6,127.9,127.6,127.5,125.5,124.6,120.5,120.2,115.6,111.8$ $110.0,99.6,71.1,66.0,53.1,-9.2$.

Palladium complex (23). Solid 20 (100 mg, $0.224 \mathrm{mmol}), \mathrm{Pd}\left(\mathrm{PPh}_{3}\right)_{4}(300 \mathrm{mg}, 0.2578$ mmol), and benzyltrimethylammonium chloride (104 mg, $0.561 \mathrm{mmol}$, dried at $70{ }^{\circ} \mathrm{C} / 0.1$ torr for $24 \mathrm{~h}$ ) were placed in a one neck $5 \mathrm{~mL}$ flask with a fused reflux condenser (one piece) and placed under vacuum (0.1 torr), purging with Ar several times. The vessel was then sealed with a septum, wrapped with parafilm, and flushed under a stream of Ar (needle inlet and outlet) for at least $15 \mathrm{~min}$. THF (degassed, $2 \mathrm{~mL}$ ) was added, the septum was then topped with vacuum grease, and the vessel heated to $70{ }^{\circ} \mathrm{C}$ (bath temp) for $12 \mathrm{~h}$. The suspension was allowed to cool and was then poured into water, turning the mixture from yellow to orange/red, and extacted with $\mathrm{Et}_{2} \mathrm{O}(2 \mathrm{X})$ and then $\mathrm{CH}_{2} \mathrm{Cl}_{2}$. The organics were then dried $\left(\mathrm{MgSO}_{4}\right)$ and concentrated. The residue was purified by flash column chromatography on silica gel (3:1 hexanes/acetone) to afford $220 \mathrm{mg}$ (94\% yield) of $\mathbf{2 3}$ as light yellow crystals; analytical tlc, 5:2 hexanes/acetone, $\mathrm{Rf}=0.32$. Pure material was obtained by crystallization from $\mathrm{CH}_{2} \mathrm{Cl}_{2} /$ hexanes, mp $160-170{ }^{\circ} \mathrm{C}$ (dec). ES Molecular ion calcd for $\mathrm{C}_{53} \mathrm{H}_{47} \mathrm{~N}_{2} \mathrm{O}_{3} \mathrm{P}_{2} \mathrm{Pd}+$ : 927.2097; found $m / z=927.2109$, error= 1 ppm; base peak= $665.1379 \mathrm{amu}\left(\right.$ loss of $\left.\mathrm{PPh}_{3}\right)$; IR (neat, $\left.\mathrm{cm}^{-1}\right) 1731(\mathrm{C}=\mathrm{O}), 1405,1153$. ${ }^{1} \mathrm{H}$ NMR $\left(500 \mathrm{MHz}, \mathrm{CDCl}_{3}, \mathrm{ppm}\right) \delta 7.43(1 \mathrm{H}, \mathrm{s}) 7.41-7.35(12 \mathrm{H}, \mathrm{m}) 7.31(1 \mathrm{H}, \mathrm{m})$ 7.28$7.22(6 \mathrm{H}, \mathrm{m}) 7.18-7.12(12 \mathrm{H}, \mathrm{m}) 7.10(1 \mathrm{H}, \mathrm{s}) 6.80-6.77(1 \mathrm{H}, \mathrm{m}) 6.33(1 \mathrm{H}, \mathrm{t}, J=7.8 \mathrm{~Hz})$ $2.41(3 \mathrm{H}, \mathrm{s}) 1.64(9 \mathrm{H}, \mathrm{s}) .{ }^{13} \mathrm{C} \mathrm{NMR}\left(100 \mathrm{MHz}, \mathrm{CDCl}_{3}, \mathrm{ppm}\right) \delta 161.2,148.9,147.2$, $146.2,136.1,134.5\left(\mathrm{t},{ }^{2} \mathrm{~J}_{\mathrm{C}-\mathrm{P}}=6.1 \mathrm{~Hz}\right), 130.9,130.7\left(\mathrm{t},{ }^{1} \mathrm{~J}_{\mathrm{C}-\mathrm{P}}=22.5 \mathrm{~Hz}\right), 129.6,127.6\left(\mathrm{t},{ }^{2} \mathrm{~J}_{\mathrm{C}-}\right.$ 
$\mathrm{P}=5.0 \mathrm{~Hz}), 124.4,123.7,122.7,111.6,109.5,83.3,77.2,28.2,14.4 .{ }^{31} \mathrm{P}$ NMR $(162$ $\left.\mathrm{MHz}, \mathrm{CDCl}_{3}, \mathrm{ppm}\right) \delta 21.4$.

Coupled product (24). Solid 19 (163 mg, $0.304 \mathrm{mmol})$ and 23 (160 mg, $0.166 \mathrm{mmol})$ were placed under vacuum ( 0.1 torr), purging with Ar several times. The vessel was then sealed with a septum, wrapped with parafilm, and flushed under a stream of $\mathrm{Ar}$ (needle inlet and outlet) for at least $15 \mathrm{~min}$. Benzene (degassed, $1.2 \mathrm{~mL}$ ) was added, the septum was then topped with vacuum grease, and the vessel heated to $65^{\circ} \mathrm{C}$ (bath temp) for $15 \mathrm{~h}$. The ensuing black suspension was cooled to $\mathrm{rt}$ and then poured into $5 \% \mathrm{NaHCO}_{3}$ solution and extracted with $\mathrm{Et}_{2} \mathrm{O}(3 \mathrm{X})$. The $\mathrm{Et}_{2} \mathrm{O}$ was then dried $\left(\mathrm{MgSO}_{4}\right)$ and concentrated. The residue was purified by flash column chromatography on silica gel (3:2 hexanes/EtOAc) eluting $\mathbf{1 9}$ in the early fractions, pure $\mathbf{2 4}$ in a few middle fractions and then mixed fractions containing $\mathbf{2 4}$ and $\mathbf{2 3}$. The mixed fractions were then concentrated and purified by preparative tlc (1:1 hexanes/EtOAc) to afford $60.5 \mathrm{mg}$ (54\% yield) of $\mathbf{2 4}$ as a white foam and $50 \mathrm{mg}$ of $\mathbf{2 3}$. Stannane $\mathbf{1 9}$ was then further purified by flash column chromatography on silica gel (9.5:1 hexanes/EtOAc) to recover $90 \mathrm{mg}$. Data for $\mathbf{2 4}$; analytical tlc, 5:2 hexanes/acetone, $\mathrm{Rf}=0.35$. ES Molecular ion calcd for $\mathrm{C}_{40} \mathrm{H}_{35} \mathrm{~N}_{3} \mathrm{O}_{7} \mathrm{Na}+$ : 692.2367 ; found $m / z=692.2377$, error= 1 ppm; IR (neat, $\mathrm{cm}^{-1}$ ) 3409 $(\mathrm{NH}), 1734(\mathrm{C}=\mathrm{O}), 1485,1153 .{ }^{1} \mathrm{H}$ NMR $\left(20{ }^{\circ} \mathrm{C}, 400 \mathrm{MHz}\right.$, benzene- $\left.\mathrm{d}_{6}, \mathrm{ppm}\right) \delta 8.64$ (minor atropisomer, 0.4H, d, $J=8.1 \mathrm{~Hz}$ ) 8.57 (major atropisomer, 1H, d, $J=8.7 \mathrm{~Hz}$ ) 7.75 (major, 1H, s) 7.74 (minor, 0.4H, s) 7.59 (major, $1 \mathrm{H}, \mathrm{d}, J=7.7 \mathrm{~Hz})$ 7.57-7.49 $(2.2 \mathrm{H}, \mathrm{m}$ ) 7.34-7.31 $(1.4 \mathrm{H}, \mathrm{m})$ 7.27-6.92 $(15.6 \mathrm{H}, \mathrm{m}) 6.71-6.61(7.3 \mathrm{H}, \mathrm{m}) 6.56$ (major, $1 \mathrm{H}, \mathrm{dd}, J=$ 8.8, 2.6 Hz) 6.30 (minor, 0.4H, s) 6.28 (major, 1H, s) 4.81 (minor, 0.8H, s) 4.66 (major, 2H, s) 4.42 (minor, 0.4H, d, $J=2.2 \mathrm{~Hz}$ ) 4.35 (major, $1 \mathrm{H}, \mathrm{d}, J=2.9 \mathrm{~Hz}$ ) 3.52 (major, 3H, 
s) $3.17(1.4 \mathrm{H}, \mathrm{s}) 1.98$ (major, $3 \mathrm{H}, \mathrm{s}) 1.53$ (minor, $1.4 \mathrm{H}, \mathrm{s}$ ) 1.37 (major, 9H, s) 1.36

(minor, $3.7 \mathrm{H}, \mathrm{s}) .{ }^{13} \mathrm{C}$ NMR $\left(20{ }^{\circ} \mathrm{C}, 100 \mathrm{MHz}\right.$, benzene- $\left.\mathrm{d}_{6}, \mathrm{ppm}\right) \delta 170.3,170.2,161.1$, 154.6, 153.8, 149.7, 146.6, 144.9, 144.8, 138.2, 136.9, 132.4, 130.5, 130.4, 129.0, 128.9, $128.5,128.3,128.2,127.9,127.6,127.2,126.5,126.1125 .8,125.6,125.2,124.5,124.2$, $122.5,119.8,119.6,116.4,116.3,115.6,115.5,112.7,112.4,111.0,110.7,110.5,101.0$ $100.6,84.4,84.2,71.5,71.3,66.9,53.1,52.8,28.2,13.7,13.2$.

Macrocycle precursor (28/29). To a solution of $24(79.7 \mathrm{mg}, 0.119 \mathrm{mmol})$ in $\mathrm{MeOH}$ (2.0 mL), THF (for solubility, $1.0 \mathrm{~mL})$, and AcOH $(0.2 \mathrm{~mL})$ was added formalin $(1.0 \mathrm{~mL}$ of 37\% aqueous). The clear colorless solution was stirred overnight and poured into 5\% $\mathrm{NaHCO}_{3}$ solution and extracted with $\mathrm{Et}_{2} \mathrm{O}(3 \mathrm{X})$. The $\mathrm{Et}_{2} \mathrm{O}$ was then dried $\left(\mathrm{MgSO}_{4}\right)$ and concentrated. Analytical tlc indicated the presence of two distinct components that were partly separable and were slowly interconverting during chromatography. The products appeared as two spots blended into a streak between $\operatorname{Rf} 0.31$ and 0.57 in 1:1 hexanes/EtOAc. Because of this, the residue could not be purified by flash column chromatography as $\mathbf{2 8 / 2 9}$ would elute over many fractions. It was determined that the residue could be purified by preparative tlc (1:1 EtOAc/hexanes). This gave ample time for $\mathbf{2 8}$ to $\mathbf{2 9}$ interconversion and produced a singe band affording $85 \mathrm{mg}$ (quant.) of $\mathbf{2 8 / 2 9}$ as a white foam. ES Molecular ion calcd for $\mathrm{C}_{42} \mathrm{H}_{39} \mathrm{~N}_{3} \mathrm{O}_{8} \mathrm{Na}+$ : 736.2629 ; found $\mathrm{m} / z=$ 736.2654, error $=3 \mathrm{ppm}$; IR (neat, $\left.\mathrm{cm}^{-1}\right) 1735(\mathrm{C}=\mathrm{O}), 1485,1153 .{ }^{1} \mathrm{H}$ NMR $\left(20^{\circ} \mathrm{C}, 500\right.$ $\mathrm{MHz}$, benzene- $\mathrm{d}_{6}, \mathrm{ppm}$ ) $\delta 8.63$ (minor atropisomer, $0.67 \mathrm{H}, \mathrm{d}, J=6 \mathrm{~Hz}$ ) 8.52 (major atropisomer, 1H, d, J=6.0 Hz) 7.85 (major, 1H, s) 7.80 (minor, $0.63 \mathrm{H}, \mathrm{s}$ ) 7.69 (minor, $0.69 \mathrm{H}, \mathrm{dd}, J=7.5,1.5 \mathrm{~Hz}$ ) 7.52-7.60 $(2.78 \mathrm{H}, \mathrm{m}) 7.37$ (major, $1 \mathrm{H}, \mathrm{dd}, J=8.0,0.5 \mathrm{~Hz}$ ) 7.25-7.17 $(5.41 \mathrm{H}, \mathrm{m})$ 7.14-7.00 $(10.2 \mathrm{H}, \mathrm{m}) 6.85$ (major, $1 \mathrm{H}, \mathrm{dd}, J=8.0,1.5 \mathrm{~Hz}) 6.78$ 
(minor, 0.80H, t, J= 7.5 Hz) 6.76-6.69 $(2.67 \mathrm{H}, \mathrm{m}) 6.63-6.59(2.37 \mathrm{H}, \mathrm{m}) 6.46$ (major, $1 \mathrm{H}$, s) 5.95 (minor, $0.69 \mathrm{H}, \mathrm{s}) 4.91$ (minor, $1.5 \mathrm{H}, \mathrm{ABq}, J=11.5 \mathrm{~Hz}$ ) 4.68 (major, $2 \mathrm{H}, \mathrm{ABq}, J=$ $11.5 \mathrm{~Hz}$ ) 4.33 (major, $2 \mathrm{H}, \mathrm{ABq}, J=10.0 \mathrm{~Hz}$ ) 3.95 (minor, $1.4 \mathrm{H}, \mathrm{ABq}, J=10.0 \mathrm{~Hz}$ ) 3.37 (major, 3H, s) 3.11 (minor, 2H, s) 2.63 (major, 3H, s) 2.58 (minor, 2.2H, s) 1.92 (major, 3H, s) 1.66 (minor, 2.1H, s) 1.32 (major, 9H, s) 1.31 (minor, 6.3H, s).

Macrocycle (30). Macrocycle precursor 28/29 (85 mg, $0.119 \mathrm{mmol}$ ) was dissolved in THF (24 mL) and cooled to $-78^{\circ} \mathrm{C}$. LDA $(0.5 \mathrm{~mL}, 0.60 \mathrm{M}$ solution in THF, $0.3 \mathrm{mmol})$ was then added in one portion and the reaction was stirred for 10 min during which time the clear colorless solution became a yellow/orange color. The reaction was quenched with $\mathrm{AcOH}(0.15 \mathrm{~mL})$, turning the solution clear colorless, and then poured into $5 \%$ $\mathrm{NaHCO}_{3}$ solution and extracted with $\mathrm{Et}_{2} \mathrm{O}(3 \mathrm{X})$. The $\mathrm{Et}_{2} \mathrm{O}$ was then dried $\left(\mathrm{MgSO}_{4}\right)$ and concentrated. The residue was purified by preparative tlc $(1: 1$ hexanes/EtOAc) to afford $23.7 \mathrm{mg}$ (29\% yield) of $\mathbf{3 0}$ as a white solid residue and $53 \mathrm{mg}$ of $\mathbf{2 8 / 2 9}$ in a lower band $(\mathrm{Rf}=0.49)$; analytical tlc, 1:1 hexanes/EtOAc, $\mathrm{Rf}=0.56 . \mathrm{ES}$ Molecular ion calcd for $\mathrm{C}_{41} \mathrm{H}_{35} \mathrm{~N}_{3} \mathrm{O}_{7} \mathrm{Na}+:$ 704.2367; found $m / z=704.2382$, error= 2 ppm; IR (neat, $\mathrm{cm}^{-1}$ ) 1742 $(\mathrm{C}=\mathrm{O}), 1715(\mathrm{C}=\mathrm{O}), 1364 ;{ }^{1} \mathrm{H} \mathrm{NMR}\left(500 \mathrm{MHz}, \mathrm{CDCl}_{3}, \mathrm{ppm}\right) \delta 8.28(1 \mathrm{H}, \mathrm{d}, J=8.6 \mathrm{~Hz})$ $7.72(1 \mathrm{H}, \mathrm{s}) 7.48-7.27(8 \mathrm{H}, \mathrm{m}) 6.98(1 \mathrm{H}, \mathrm{dd}, J=7.5,1.3 \mathrm{~Hz}) 6.79(1 \mathrm{H}, \mathrm{dd}, J=8.6,2.7 \mathrm{~Hz})$ $6.74(1 \mathrm{H}, \mathrm{s}) 6.73(1 \mathrm{H}, \mathrm{d}, J=9 \mathrm{~Hz}) 6.69(1 \mathrm{H}, \mathrm{dd}, J=1.3,7.5 \mathrm{~Hz}) 6.62(1 \mathrm{H}, \mathrm{t}, J=7.5 \mathrm{~Hz})$ $6.50(1 \mathrm{H}, \mathrm{s}) 5.05(2 \mathrm{H}, \mathrm{ABq}, J=11.5 \mathrm{~Hz}) 4.33(2 \mathrm{H}, \mathrm{ABq}, J=10.2 \mathrm{~Hz}) 4.07(2 \mathrm{H}, \mathrm{ABq}, J=$ $15.0 \mathrm{~Hz}) 2.70(3 \mathrm{H}, \mathrm{s}) 1.70(9 \mathrm{H}, \mathrm{s}) .{ }^{13} \mathrm{C} \mathrm{NMR}\left(125 \mathrm{MHz}, \mathrm{CDCl}_{3}, \mathrm{ppm}\right) \delta 197.4,156.2$, $154.1,152.5,149.4,144.9,144.3,137.4,134.9,133.0,132.4,130.3,128.8,128.6,128.2$ $128.1,127.9,127.2,126.8,125.4,124.8,123.1,120.3,119.4,116.1,115.2,113.6,110.3$ $108.4,102.2,85.1,77.6,71.4,70.9,54.6,42.0,28.4$. 
Acetamide (32). Macrocycle 30 (10 mg, $0.015 \mathrm{mmol})$ was dissolved in THF (0.1 mL) and cooled to $-78{ }^{\circ} \mathrm{C}$. KHMDS $(0.05 \mathrm{~mL}, 0.01614 \mathrm{mmol})$ was added slowly down the side of the flask (to prevent warming). The solution darkened to a yellow/orange upon addition. Aminating reagent $\mathbf{3 1}(8.3 \mathrm{mg}, 0.022 \mathrm{mmol})$ was added as a solution in THF $(0.1 \mathrm{~mL})$ slowly down the side of the flask. The reaction stirred for $3 \mathrm{~h}$ over which time the solution became dark green. Acetic anhydride $(0.05 \mathrm{~mL})$ was added and the cold bath was removed. Once at $\mathrm{rt}$ the solution was poured into a sat'd $\mathrm{NaHCO}_{3}$ solution and extracted with $\mathrm{Et}_{2} \mathrm{O}(2 \mathrm{X})$. The $\mathrm{Et}_{2} \mathrm{O}$ was then dried $\left(\mathrm{Mg}_{2} \mathrm{SO}_{4}\right)$ and concentrated giving a residue that was purified by by preparative tlc (1:1 hexanes/EtOAc) to afford $1.5 \mathrm{mg}$ (14\% yield) of $\mathbf{3 2}$ as a white solid residue and $4 \mathrm{mg}$ of recovered $\mathbf{3 0}$ in a higher band $(\mathrm{Rf}=0.64)$; analytical tlc, 1:1 hexanes/EtOAc, $\mathrm{Rf}=0.20$. ES Molecular ion calcd for $\mathrm{C}_{43} \mathrm{H}_{38} \mathrm{~N}_{4} \mathrm{O}_{8} \mathrm{Na}+:$ 761.2; found $m / z=761.2 ;{ }^{1} \mathrm{H}$ NMR (500 MHz, benzene- $\mathrm{d}_{6}, \mathrm{ppm}$ ) $\delta 8.50(1 \mathrm{H}, \mathrm{d}, J=8.5 \mathrm{~Hz}) 7.90(1 \mathrm{H}, \mathrm{d}, J=2.5 \mathrm{~Hz}) 7.39(1 \mathrm{H}, \mathrm{s}) 7.28-7.13(6 \mathrm{H}, \mathrm{m}) 7.10-$ $7.04(2 \mathrm{H}, \mathrm{m}) 7.02(1 \mathrm{H}, \mathrm{dd}, J=7.6,1.4 \mathrm{~Hz}) 6.78(1 \mathrm{H}, \mathrm{d}, J=7.6 \mathrm{~Hz}) 6.64-6.59(2 \mathrm{H}, \mathrm{m})$ $6.54(1 \mathrm{H}, \mathrm{dd}, J=7.6,1.4 \mathrm{~Hz}) 6.41(1 \mathrm{H}, \mathrm{t}, J=7.6 \mathrm{~Hz}) 6.38(1 \mathrm{H}, \mathrm{s}) 6.07(1 \mathrm{H}, \mathrm{d}, J=7.6 \mathrm{~Hz})$ $4.77(2 \mathrm{H}, \mathrm{ABq}, J=11.5 \mathrm{~Hz}) 4.27(2 \mathrm{H}, \mathrm{ABq}, J=10.5 \mathrm{~Hz}) 2.69(3 \mathrm{H}, \mathrm{s}) 1.44(3 \mathrm{H}, \mathrm{s}) 1.31$ $(9 \mathrm{H}, \mathrm{s})$.

Bisoxazole (33). Pyridine $(2 \mathrm{~mL})$ and freshly distilled $\mathrm{POCl}_{3}(1 \mathrm{~mL})$ were mixed. Once homogenous, $0.4 \mathrm{~mL}$ of this solution was added to acetamide $32(2.5 \mathrm{mg}, 0.0034 \mathrm{mmol})$ and heated to $70{ }^{\circ} \mathrm{C}$ for $6 \mathrm{~h}$. The ensuing dark brown suspension was cooled to $\mathrm{rt}$ and poured into a rapidly stirred solution of $\mathrm{NaHCO}_{3}$ (sat'd). The resulting mixture was extracted with $\mathrm{Et}_{2} \mathrm{O}(3 \mathrm{X})$. The $\mathrm{Et}_{2} \mathrm{O}$ was then washed sequentially with $0.1 \mathrm{M} \mathrm{H}_{2} \mathrm{SO}_{4}$ and 
sat'd $\mathrm{NaHCO}_{3}$ sol'n, dried $\left(\mathrm{MgSO}_{4}\right)$ and concentrated. The residue was purified by preparative tlc (3:1 hexanes/acetone) to afford $1.1 \mathrm{mg}$ (ca. 40\%) of $\mathbf{3 3}$ as a white solid residue and $1.2 \mathrm{mg}$ (ca. $50 \%$ ) of $\mathbf{3 4}$ in a lower band $(\mathrm{Rf}=0.17)$; analytical tlc, 3:1 hexanes/acetone, $\mathrm{Rf}=0.21$. ES Molecular ion calcd for $\mathrm{C}_{41} \mathrm{H}_{32} \mathrm{~N}_{4} \mathrm{O}_{6} \mathrm{Na}$ : 669.2 ; found $m / z=669.2 ;{ }^{1} \mathrm{H}$ NMR $\left(500 \mathrm{MHz}\right.$, benzene- $\left.\mathrm{d}_{6}, \mathrm{ppm}\right) \delta 8.55(1 \mathrm{H}, \mathrm{d}, J=8.5 \mathrm{~Hz}) 7.50(1 \mathrm{H}$, s) 7.28-7.24 (4H, m) 7.18-7.05 (4H, m) $6.79(1 \mathrm{H}, \mathrm{dd}, J=7.6,1.4 \mathrm{~Hz}) 6.78(1 \mathrm{H}, \mathrm{d}, J=9.1$ Hz) $6.76(1 \mathrm{H}, \mathrm{s}) 6.74(1 \mathrm{H}, \mathrm{dd}, J=1.4,7.6 \mathrm{~Hz}) 6.64(1 \mathrm{H}, \mathrm{dd}, J=9.1,3.0 \mathrm{~Hz}) 6.48(1 \mathrm{H}, \mathrm{t}$, $J=7.6 \mathrm{~Hz}) 6.16(1 \mathrm{H}, \mathrm{d}, J=4.1 \mathrm{~Hz}) 4.70(2 \mathrm{H}, \mathrm{ABq}, J=11.5 \mathrm{~Hz}) 4.58(1 \mathrm{H}, \mathrm{d}, J=4.1 \mathrm{~Hz})$ $1.88(3 \mathrm{H}, \mathrm{s}) 1.36(9 \mathrm{H}, \mathrm{s})$.

Added reference 13: Ma, D. W.; Wu, Q. Q.; Terahedron Lett. 2000, 41, 9089. 
